\title{
A New Approach for Enhancing Image Retrieval using Neutrosophic Sets
}

\author{
Mohamed Eisa \\ Computer Science Department, \\ Port Said University, 42526, Egypt
}

\begin{abstract}
This paper adopts a novel model for Content-Based Image Retrieval (CBIR) system depending on an excellent segmentation strategy and combination of Visual Descriptors (VDs). The presented model is divided into four main phases: image segmentation, visual descriptors, Dimensionality Reduction (DR) and similarity matching. An improved segmentation technique based on Neutrosophic Sets (NSs) is proposed and applied to see their ability and accuracy to segment images. In relative to the VDs, the geometrical moments are used to extract the shape of an object, the modified Stricker method to the color extraction is proposed and the MPEG-7 edge histogram descriptor is presented for each of them. Experimental results presented show that the proposed model provides precise image retrieval in a short time.
\end{abstract}

\section{Keywords}

NSs, GMs, EHD, Evaluation function, ANMRR

\section{INTRODUCTION}

CBIR systems retrieve relevant images in a database using visual contents of the images [1]. Advanced research is mainly developed based on the high-level semantic analysis of the image contents along with the visual contents of the image such as shape, color and edges [2]. The local image areas of interest are easily left unnoticed as the global features do not contain enough information for local discrimination [3]. A way to pay attention to local properties is to use image segmentation.

Neutrosophy is a branch of philosophy, introduced by F. Smarandache in 1995; it can solve some problems that cannot be solved by the fuzzy logic. It studies the Neutrosophic Logical (NL) values of the propositions. Each proposition is estimated to have three components: the percentage of truth in a subset $\mathrm{T}$, the percentage of indeterminacy in a subset $\mathrm{I}$ and the percentage of falsity in a subset F [4]. NL is so new that its use in many fields merits exploration. In this paper, NL is applied to the field of segmentation [5].

Define $\langle A\rangle$ as an event or entity, $\langle$ Non-A $\rangle$ is not $\langle A\rangle$ and $\langle$ Anti-A $\rangle$ is the opposite of $\langle A\rangle$. Also, $\langle$ Neut-A $\rangle$ is defined as neither $\langle A\rangle$ nor $\langle$ Anti-A $\rangle$. For example, if $\langle A\rangle=$ white, then $\langle$ Anti-A $\rangle=$ black. $\langle$ Non-A $\rangle=$ blue, yellow, red, black, ... etc. (any color except white). $\langle$ Neut-A $\rangle=$ blue, yellow, red, ... etc. (any color except white and black). According to this theory every idea $\langle\mathrm{A}\rangle$ tends to be neutralized and balanced by $\langle$ Anti-A $\rangle$ and $\langle$ Non-A $\rangle$ ideas [6].

In this paper, the new NS approach based on adaptive threshold is proposed to segment images. The image is transformed into Neutrosophic Domain (ND). Each pixel in the ND can be represented as T, I and F which means the pixel is $\mathrm{t} \%$ true, $\mathrm{i} \%$ indeterminate and $\mathrm{f} \%$ false, where $\mathrm{t}$ varies in $T$, $i$ varies in $I$, and $f$ varies in $F$, respectively. A new operation is employed to reduce the indetermination degree of the image, which is evaluated by the entropy of the indeterminate subset. Then, the image becomes more uniform and homogenous, and more suitable for thresholding.

A common scheme to represent the image is to extract different types of VDs such as shape, color and edges. Moment functions of the two-dimensional image intensity distribution are used in a variety of applications, as descriptors of shape. Image moments that are invariant with respect to the transformations of scale, translation, and rotation find applications in areas such as pattern recognition, object identification and template matching [7].

In general, histogram-based methods provide overall image characteristics. However, they show high sensitivity to illumination and cannot represent localized features well. An advanced color extraction method to compensate the above drawbacks of histogram-based methods is proposed [8].

Edges in images constitute an important feature to represent their contents. An edge histogram in the image space represents the frequency and the directionality of the brightness changes in the image. It is a unique feature for images, which cannot be duplicated by a color histogram or the texture features [9]. The edge histogram descriptor expresses only the local edge distribution in the image which may not be sufficient to represent global features of the edge distribution. So the semi-global and global edge histograms from the local histogram bins are presented.

Although the integration of multi features enhances the image detection rate and increases the image retrieval performance, but the speed of the content-based image retrieval is slower mainly due to the curse of high dimensionality of the feature space. Therefore, to enhance image detection rate and simplify computation of image retrieval, a DR technique must be adopted such as feature selection [10]. Feature selection is a one solution for computational cost problem of CBIR. It refers to selecting the most important features and their combinations for describing and querying items in the database in order to reduce retrieval complexity in terms of process time while maintaining high retrieval performance.

The rest of this paper is composed of the following sections. Section 2 shows the proposed new NS for segment image. The VDs is introduced in section 3. DR algorithm is outlined in section 4 . The similarity matching for each VD is presented separately and also for combined VDs in section 5. Experimental results are shown in section 6. Finally, the conclusion is presented in section 7 .

\section{SEGMENTATION}

2.1 Neutrosophic Set (NS)

NS and its properties are discussed briefly in [11].

Definition 1 (NS): define $\mathrm{T}$, I and $\mathrm{F}$ as neutrosophic components to represent $\langle\mathrm{A}\rangle,\langle$ Neut-A $\rangle$ and $\langle$ Anti-A $\rangle$. Let $\mathrm{T}, \mathrm{I}$ and $\mathrm{F}$ be standard or non-standard real subsets of $]-0,1+[$ 
with $\sup \mathrm{T}=\mathrm{t} \_$sup, inf $\mathrm{T}=\mathrm{t} \_$inf, $\sup \mathrm{I}=\mathrm{i} \_$sup, inf $\mathrm{I}=\mathrm{i} \_$inf, $\sup F=f \_s u p, \inf F=f \_i n f$ and $n \_s u p=t \_s u p+i \_s u p+f \_s u p$, $\mathrm{n} \_$inf $=\mathrm{t} \_$inf $+\mathrm{i} \_$inf $+\mathrm{f} \_$inf. $\mathrm{x} \_$sup specifies the superior limits of the subsets, and $x \_$inf specifies the inferior limits of the subsets. T, I and F are not necessarily intervals, but may be any real subunitary subsets. T, I and F are set-valued vector functions or operations depending on known or unknown parameters and they may be continuous or discrete. Additionally, they may overlap or be converted from one to the other. An element $A(T, I, F)$ belongs to the set in the following way: it is true $(t \in T)$, i indeterminate $(i \in I)$ and $\mathrm{f}$ false $(f \in F)$ where $\mathrm{t}, \mathrm{i}$, and $\mathrm{f}$ are real numbers in the sets $\mathrm{T}$, I and $\mathrm{F}$. In a classical set, $\mathrm{I}=\boldsymbol{Q}$, inf $\mathrm{T}=\sup \mathrm{T}=0$ or 1 , inf $F=\sup F=0$ or 1 and $\sup T=\sup F=1$. In a fuzzy set, $I=Q$, $\inf \mathrm{T}=\sup \mathrm{T}=[0,1], \inf \mathrm{F}=\sup \mathrm{F}=[0,1]$ and $\sup \mathrm{T}=\sup \mathrm{F}$ $=1$. In a NS, $\boldsymbol{I}, \boldsymbol{T}, \boldsymbol{F} \in] \mathrm{O},+1[$ [12]. In order to apply neutrosophy, an image needs to be transferred to a ND. A pixel in the ND can be represented as T, I and F meaning the pixel is $\mathrm{t} \%$ true, $\mathrm{i} \%$ indeterminate and $\mathrm{f} \%$ false, where $\mathrm{t}$ varies in $\mathrm{T}$, $\mathrm{i}$ varies in $\mathrm{I}$ and $\mathrm{f}$ varies in $\mathrm{F}$, respectively. In a classical set, $\mathrm{i}=0, \mathrm{t}$ and $\mathrm{f}$ are either 0 or 100 . In a fuzzy set, $\mathrm{i}=0$, $\mathrm{O} \leq t, f \leq 100$. In a NS, $\mathrm{O} \leq t, i, f \leq 100$.

\subsection{Neutrosophic Image (NI)}

Definition 2 (NI): Let $\mathrm{U}$ be a universe of discourse and $\mathrm{W}$ be a set included in $\mathrm{U}$, which is composed by bright pixels. A NI $\mathrm{P}_{\mathrm{NS}}$ is characterized by three subsets $\mathrm{T}$, I and $\mathrm{F}$. A pixel $\mathrm{P}$ in the image is described as $\mathrm{P}(\mathrm{T}, \mathrm{I}, \mathrm{F})$ and belongs to $\mathrm{W}$ in the following way: it is $\mathrm{t} \%$ true in the bright pixel set, $\mathrm{i} \%$ indeterminate and $\mathrm{f} \%$ false, where $t$ varies in $\mathrm{T}$, $\mathrm{i}$ varies in $\mathrm{I}$ and $f$ varies in $F$. The pixel $P(i, j)$ in the image domain is transformed into ND $P_{N S}(i, j)=\{T(i, j), I(i, j), F(i, j)\}$. Where $T(i, j), I(i, j)$ and $F(i, j)$ are the probabilities belong to white set, indeterminate set and non-white set, respectively, which are defined as [13]:

$$
\begin{aligned}
& P_{N S}(i, j)=\{T(i, j), F(i, j), I(i, j)\} \\
& T(i, j)=\frac{\bar{g}(\overline{i, j})-\bar{g}_{\min }}{\bar{g}_{\max }-\bar{g}_{\min }} \\
& I(i, j)=1-\frac{H_{\mathrm{o}}(i, j)-H_{o}}{H_{\mathrm{O}_{\max }}-H_{\mathrm{O}_{\min }}} \\
& F(i, j)=1-T(i, j) \\
& H_{\mathrm{o}}(i, j)=\operatorname{abs}(g(i, j)-\bar{g}(\bar{i}, \bar{j}))
\end{aligned}
$$

Where $\bar{g}(\bar{i}, j)$ is the local mean value of the pixels of the window size, and $\mathrm{H}_{\mathrm{o}}(\mathrm{i}, \mathrm{j})$ is the homogeneity value of $\mathrm{T}$ at (i, $\mathrm{j}$ ), which is described by the absolute value of difference between intensity $\mathrm{g}(\mathrm{i}, \mathrm{j})$ and its local mean value $\bar{g}(\bar{i}, j)$. After the image is transformed in NS domain, some concepts and operations in ND are defined and employed.

\subsection{Entropy of NI}

Entropy is utilized to evaluate the distribution of different gray level in images. If the entropy is maximum, the different intensities have equal probability and the intensities distribute uniformly. If the entropy is small, the intensities have different probabilities and their distributions are non-uniform [14].
Definition 3 (NI entropy): NI entropy is defined as the summation of the entropies of three subset T, I and F, which is employed to evaluate the distribution of the elements in ND:

$$
\begin{aligned}
& E n_{T}=-\sum P_{T}(i) \ln P_{T}(i) \\
& E n_{F}=-\sum P_{F}(i) \ln P_{F}(i) \\
& E n_{I}=-\sum P_{I}(i) \ln P_{I}(i) \\
& E n_{N S}=E n_{T}+E n_{F}+E n_{I}
\end{aligned}
$$

Where, $E n_{T}, E n_{I}$ and $E n_{F}$ are the entropy of subsets $T, I$ and $F$ respectively. $P_{T}(i), P_{I}(i)$ and $P_{F}(i)$ are the probabilities of element i in $T, I$ and $F$, respectively. $E n_{T}$ and $E n_{F}$ are utilized to measure the distribution of the elements in NS and the entropy of $T, E n_{I}$ is employed to evaluate the indeterminacy distribution.

\subsection{Adaptive Threshold}

After the true subset $\mathrm{T}$ is handled and the noise in $\mathrm{T}$ is removed and $\mathrm{T}$ becomes more homogeneous, which is suitable to segment the subset $\mathrm{T}$ precisely even using adaptive threshold method. Local adaptive threshold used to select an individual threshold for each pixel based on the range of intensity values in its mean of local neighborhood. This approach is used for finding the local threshold to statistically examine the intensity values of the local neighborhood of each pixel. This method is simple, fast and less computationally intensive and produces good results for segment images [15], and the following algorithm shows the steps of the proposed approach for segmentation in details.

Algorithm 1: NL and Adaptive Threshold

1: Transfer image from image domain to ND using NL.

2: Each pixel in neutrosophic image PNS is represented by three subsets T, I and F. $P_{N S}(i, j)=\{T(i, j), I(i, j), F(i, j)\}$.

3: Calculate histogram of the image.

4: Calculate local maxima of the histogram and calculate the mean of local maxima.

5: Find the peaks greater than mean of local maxima.

6: Lets the first peak be $g_{\text {min }}$ and the last peak be $g_{\text {max }}$.

7: Calculate $\mathrm{T}(\mathrm{i}, \mathrm{j})$ from local of mean window, $g_{\text {min }}$, and $g_{\text {max }}$.

8: Calculate $I(i, j)$ from the homogeneity value of $T$ using the absolute value of difference between intensity $g(i, j)$ and its local mean value.

9: Calculate $F(i, j)=1-T(i, j)$.

10: After the image is transformed in NS domain. Entropy $\boldsymbol{E} \boldsymbol{n}_{N S}$ is utilized to evaluate the NS image for T, I and F.

11: Sum the image entropy $E n_{N S}=E n_{T}+E n_{I}+E n_{F}$

12: Apply adaptive local threshold used the mean of the local intensity distribution with static coefficient factor for slices.

13: The output from adaptive threshold is binary image. Find connected components.

\section{VISUAL DESCRIPTORS 3.1 Geometrical Moments}

The shape of an object is a very important character in human's perception, recognition, and comprehension. Because geometric shape represents the essential characteristic of an object, and has invariance with respect to translation, scale and orientation, the analysis and discernment 
like geometry are of important significance in computer vision [16].

$$
\begin{aligned}
\mathbf{M}_{1}= & \mu_{20}+\mu_{02} \\
\mathbf{M}_{2}= & \left(\mu_{20}-\mu_{02}\right)^{2}+4 \mu_{11}^{2} \\
\mathbf{M}_{3}= & \left(\mu_{30}-3 \mu_{12}\right)^{2}+3\left(\mu_{21}+\mu_{03}\right)^{2} \\
\mathbf{M}_{4}= & \left(\mu_{30}+\mu_{12}\right)^{2}+\left(\mu_{21}+\mu_{03}\right)^{2} \\
M_{5}= & \left(\mu_{30}-3 \mu_{12}\right)\left(\mu_{30}+\mu_{12}\right)\left[\begin{array}{l}
\left(\mu_{30}+\mu_{12}\right)^{2} \\
-3\left(\mu_{21}+\mu_{03}\right)^{2}
\end{array}\right] \\
& +\left(3 \mu_{21}-\mu_{03}\right)\left(\mu_{21}+\mu_{03}\right)\left[\begin{array}{l}
3\left(\mu_{30}+\mu_{12}\right)^{2} \\
-\left(\mu_{21}+\mu_{03}\right)^{2}
\end{array}\right] \\
M_{6}= & \left(\mu_{20}-\mu_{02}\right)\left[\left(\mu_{30}+\mu_{12}\right)^{2}-\left(\mu_{21}+\mu_{03}\right)^{2}\right] \\
& +4 \mu_{11}\left(\mu_{30}+\mu_{12}\right)\left(\mu_{21}+\mu_{03}\right) \\
M_{7}= & \left(3 \mu_{21}-\mu_{03}\right)\left(\mu_{30}+\mu_{12}\right)\left[\begin{array}{l}
\left(\mu_{30}+\mu_{12}\right)^{2} \\
-3\left(\mu_{21}+\mu_{03}\right)^{2}
\end{array}\right] \\
& +\left(3 \mu_{12}-\mu_{30}\right)\left(\mu_{21}+\mu_{03}\right)\left[\begin{array}{l}
3\left(\mu_{30}+\mu_{12}\right)^{2} \\
-\left(\mu_{21}+\mu_{03}\right)^{2}
\end{array}\right]
\end{aligned}
$$

Geometric moments of a $1 \mathrm{D}$ signal $\mathrm{S}(\mathrm{x})$ are defined as the following [16]:

$$
M_{n}(x)=\int_{-\omega}^{\omega} S(x+t) t^{n} d t \quad n=0,1,2, \ldots
$$

Where $M_{n}(x)$ is the moment of order n calculated from a window of size $(2 \omega+1)$ pixels centered at the point $x$. Geometric moments of a 2D image $\mathbf{I}(\mathrm{x}, \mathrm{y})$ are defined as the following [16]:

$M_{m, n}(x, y)=\int_{-\infty l}^{\sigma l} \int_{-\pi 2}^{\sigma^{2}} I(x+u, y+v) u^{m} v^{n} d u d v m, n=0,1,2, \ldots$

Where $M_{m, n}(x)$ is the moment of order $(\mathrm{m}, \mathrm{n})$ calculated from a window of size $\left(2 \varpi_{1}+1\right) \times\left(2 \varpi_{2}+1\right)$ pixels centered at the point $(\mathrm{x}, \mathrm{y})$.

\subsection{Color Feature Extraction}

There have been researches using color feature as a query key for image retrieval in general, they use color histogram methods to obtain color feature information from an image. Color histogram indexing was introduced by Swain et al. [17]. They used the brightness values of RGB color space and stored coarsely quantized color histogram of images as indices. Funt et al. modified Swain's method to improve its performance by using the histogram of the RGB color ratio from neighboring locations [18]. In general, histogram-based methods provide overall image characteristics. However, they show high sensitivity to illumination and cannot represent localized features well. An advanced color extraction method to compensate the above drawbacks of histogram-based methods is proposed.

First, when a query image is presented its RGB color space is converted into HSI (Hue, Saturation and Intensity) for better retrieval performance. Next, each image is divided into subblocks for considering spatial location information. Then, we can get a color feature vector from each sub-block.

\subsubsection{Color Feature Vector Extraction}

Stricker proposed a color extraction method using the three moments of each color channel of an image: average, standard deviation and skewness [19]. For the feature vector extraction, its color features are computed by the following equations:

$$
\begin{aligned}
& E_{i}=\frac{1}{m \cdot n} \sum_{j=1}^{m \cdot n} P_{i j} \sigma_{i}=\left[\frac{1}{m \cdot n} \sum_{j=1}^{m \cdot n}\left(P_{i j}-E_{i}\right)^{2}\right]^{\frac{1}{2}}, \\
& \alpha_{i}=\left[\frac{1}{m \cdot n} \sum_{j=1}^{m \cdot n}\left(P_{i j}-E_{i}\right)^{3}\right]^{\frac{1}{3}}
\end{aligned}
$$

Where $E_{i}$ is an average of each color channel $(i=H, S, I)$, $\sigma_{i}$ is a standard deviation, and $\alpha_{i}$ is skewness. $\boldsymbol{P}_{i j}$ is the value of each color channel at the j-th image pixel. $m \cdot n$ is a total number of pixels per image. Stricker used the 9 feature vector which consists of three moments for each color channel $\mathrm{H}, \mathrm{S}$, I respectively. A modification to the color extraction algorithm proposed by Stricker is proposed to improve the performance as follows:

-To use the color feature vectors considering spatial location information, Stricker's method to each sub-block is applied;

-The number of bins for the index in S and I channel is reduced to 2 while keeping the 16 bins for index in $\mathrm{H}$ channel, because $\mathrm{S}$ and I do not much affect the retrieval performance directly,

-Only, the first moment for $\mathrm{S}$ and I channels except for $\mathrm{H}$ is used, for which also, the second and third moments are calculated. Therefore, 5 color features are used instead of Stricker's 9 features. For the k-th $m \cdot n$ sub-block, we use the 5 features vector: The average $E_{k, H}$, standard deviation $\left(\sigma_{k, H}\right)$ and skewness $\left(\alpha_{k, H}\right)$ for $\mathrm{H}$ channel, and the average $\left(E_{k, S}, E_{k, I}\right)$ for $\mathrm{S}$ and I channels respectively.

\subsection{MPEG-7 Edge Histogram Descriptor}

\subsubsection{Definition and Semantics}

The EHD in MPEG-7 represents local edge distribution in the image. Specifically, dividing the image space into $4 \times 4$ subimages, the local-edge distribution for each sub-image can be represented by a histogram. To generate the histogram, edges in the sub-images are categorized into five types; vertical, horizontal, 45-degree diagonal, 135-degree diagonal and nondirectional edges. Since there are 16 sub-images, a total of $5 \times 16=80$ histogram bins are required. Table 1 summarizes the semantics of the 80-bin EHD [20].

Table 1. Semantics of the histogram bins of the EHD

\begin{tabular}{ll}
\hline Histogram bins & \multicolumn{1}{c}{ Semantics } \\
\hline BinCounts[0] & Vertical edge of sub-image at $(0,0)$ \\
BinCounts[1] & Horizontal edge of sub-image at $(0,0)$ \\
BinCounts[2] & 45-degree edge of sub-image at $(0,0)$ \\
BinCounts[3] & 135-degree edge of sub-image at $(0,0)$ \\
BinCounts[4] & nondirectional edge of sub-image at $(0,0)$ \\
BinCounts[5] & Vertical edge of sub-image at $(0,1)$ \\
& $\cdot$ \\
& \\
BinCounts[78] & 135-degree edge of sub-image at $(3,3)$ \\
BinCounts[79] & nondirectional edge of sub-image at $(3,3)$ \\
\hline
\end{tabular}

\subsubsection{Extraction of Edge Histogram}

For the EHD, it is required to detect nondirectional edges as well as four directional edges. The nondirectional edges include the edges with no particular directionality. The five types of edges can be extracted by a block-based edge extraction scheme. To do that, each sub-image is further divided into nonoverapping square image-blocks. The size of the image-block is dependent on the image resolution. Regardless of the size of the given image, the image space is divided into a predetermined number of image-blocks.

Each of the image-blocks is then classified into one of the five edge categories mentioned above or as a non edge block. A simple method to do this classification is to treat each image- 
block as a $2 \times 2$ super-pixel image-block and apply appropriate oriented edge detectors (Fig. 1) to compute the corresponding edge strengths. The edge detector with maximum edge strength is then identified. If this edge strength is above a given threshold, then the corresponding edge orientation is associated with the image-block. If the maximum of the edge strengths is below the given threshold, then that block is not classified as an edge block. The global edge histogram and some semi-global edge histograms are generated directly from BinCounts[i], $\mathrm{i}=0, \ldots 79$. The global histogram represents the edge distribution for the whole image space. Since there are 5 edge types, the global edge histogram has 5 bins and each bin value is obtained by accumulating and normalizing the dequantized bin values of the corresponding edge type of BinCounts[]. Consequently, we have a total of 10 bins (5 bins (local) +5 bins (global)) for the similarity matching.

\begin{tabular}{|l|l|l|l|l|l|l|l|l|l|l|}
\hline 1 & -1 & 1 & 1 & $\sqrt{2}$ & 0 & 0 & $\sqrt{2}$ & 2 & -2 \\
\hline 1 & -1 & -1 & -1 & 0 & $-\sqrt{2}$ & $-\sqrt{2}$ & 0 & -2 & 2 \\
\hline
\end{tabular}

Fig 1: Filter coefficients for edge detection

\section{DIMENSIONALITY REDUCTION ALGORITHMS}

The VDs extracted are so large and consume the time in images retrieval. A proposed solution to this problem is to classify the original VDs into relevant and irrelevant VDs according to their correlation with target and correlation with each other. A concise algorithm to perform the VDs classification is shown in figure 2.

For $i=1$ to $N$

Calculate $/ C\left(A_{i}, T\right) /$

If $\left(C\left(A_{i}, T\right) \leq \varepsilon l\right.$

Then

Drop VD $A_{i}$ from VDs set

Else

For $j=1$ to $N$

Calculate $/ C\left(A_{i}, A_{j}\right) / i \neq j$

If $\left(C\left(A_{i}, A_{j}\right) \leq \varepsilon 2\right.$

Then

Put VD $A_{i}$ in the best VDs set

Else

Ignore $A_{j}$ from the original VDs set ) $N e x t j$

)

\section{Fig 2: VDs Classification Algorithm}

Where; N: Total number of VDs in the original database table. $\varepsilon_{1}$ : Threshold level for ignoring the VDs according to their correlation with target. $\varepsilon_{2}$ : Threshold level for ignoring the VDs according to their correlation with each other. $C\left(A_{i}, T\right)$ : The correlation between the VD, i and target. $C\left(A_{i}, A_{j}\right)$ : The correlation between the VD, $i$ and $\mathrm{VD}, \mathrm{j}$.

In order to simplify the application of this algorithm on real images database, it is necessary to perform the following steps;

-Detect the numerical VD(s) in the given database table and transform it into linguistic terms.

-Check the dominant values in each VD for dropping unnecessary VD(s).

-Choose the most relevant set of $\mathrm{VD}(\mathrm{s})$ by the calculation of the conditional probability and the correlation between VD to class and VD to VD.

\subsection{Fuzzification Algorithm}

This process transfers the continuous VD values into linguistic terms. This test makes the algorithm deals with a few numbers of linguistic terms instead of a huge number of continuous values. Therefore, the search space is reduced [21]. Linguistic variables may be introduced in several ways. If the input $x \in X$ is given as a real number or a large number of integers linguistic variables are created dividing the data range $\mathrm{X}$ into distinct (for crisp logic) sets Xi. A typical linguistic variables associated with the person tall, attribute will be short if $x<1.5 m$, normal if $1.5 \leq x \leq 1.8$ and high if $x>1.8$. In this paper to avoid the problem of overlapping, a non-overlapping rectangular membership functions are used and the bounds of each linguistic term are determined by using the smooth histogram of real values.

\subsection{Features Reduction Via Dominant Values Test}

Real images can have universal VDs set $\mathrm{U}_{\text {att }}$. $U_{\text {att }}=C_{n o} \cup C_{o n} \cup C_{a l}$

Where;

$\mathrm{C}_{\mathrm{no}}$ : VDs with normal values (The VD contains a small number of values with approximately the same distribution such as (high, normal, low,....etc).

$\mathrm{C}_{\mathrm{on}}$ : VDs with one value (All the values are uniformly something such as "normal" or "low" or "high". They do not contain any information that helps to distinguish between different rows. Because they lack any information content, they should be ignored).

$\mathrm{C}_{\mathrm{al}}$ : VDs with almost only one value (Almost all the records have the same value for that VD. As a general rule of thumb, if 85-99 percent of the values in the VD are identical, the VD is likely to be useless).

Any given VD in $\mathrm{U}_{\text {att }}$ may have $\boldsymbol{n}$ values; $\left\{v_{1}, v_{2}, \ldots, v_{k}, \ldots v_{n}\right\}$. These values can be used as a measure for determining the VD type by calculating their probabilities of existence $\left\{P_{1}, P_{2}, \ldots, P_{k}, \ldots P_{n}\right\}$. If $\mathrm{p}_{\mathrm{k}}=1.0$, then the $\mathrm{VD} \in \mathrm{C}_{\mathrm{on}}$, If $0.85 \leq \mathrm{p}_{\mathrm{k}}<$ 1.0 Then the VD $\in \mathrm{C}_{\mathrm{al}}$, Such VDs must be dropped from $\mathrm{U}_{\mathrm{att}}$ because they have trivial information [22]. Figure 3 shows the algorithm for this methodology.

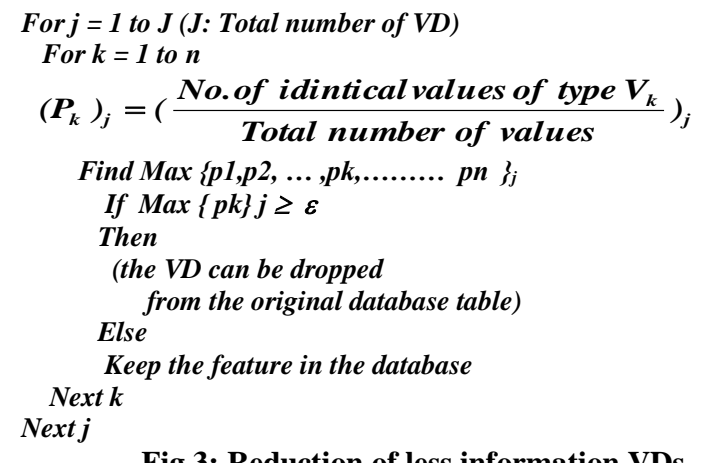

\subsection{Most Relevant VDs Selection}

The preliminary pruning of unnecessary VDs had been performed via the dominant value test algorithm. So a new universal VD set, $\mathrm{U}_{\text {att_new }}\left(\mathrm{U}_{\text {att_new }} \subseteq \mathrm{U}_{\text {att }}\right)$ is constructed. This set is now suitable for the extraction of the most relevant VDs, $\mathrm{U}_{\text {att_most }},\left(\mathrm{U}_{\text {att_most }} \subseteq \mathrm{U}_{\text {att_new }}\right)$. 


\subsubsection{Evaluation function}

An evaluation function, $\left(E_{S}\right)$, presented in [23] was used to determine whether the feature may be included in the most relevant features set or not. This function is given by:

$$
E_{S}=\frac{n * C_{A T}}{\sqrt{n+n(n-1) * C_{A A}}},
$$

Where;

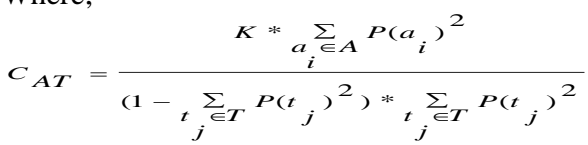

$$
\begin{aligned}
& k=\left[\sum_{t, j} \in T\left(t_{j}\right) *(1-P(t))\right]- \\
& \sum_{i} \in A\left\{\frac{P\left(a_{i}\right)^{2}}{\sum_{a_{i} \in A} P\left(a_{i}\right)^{2}}{ }_{t} \sum_{j} \in T\left(t_{j} \mid a_{i}\right) *\left(1-P\left(t_{j} \mid a_{i}\right)\right)\right\}
\end{aligned}
$$

$t_{j}$ : All possible values of target T. $a_{i}$ : All possible values of VD A. $n$ : Number of VD in $U_{\text {att new }} C_{A A}$ : average of VD-VD corrélations. $\mathrm{C}_{\mathrm{AT}}$ : average of VD-target correlations. $\mathrm{P}\left(\mathrm{a}_{\mathrm{i}}\right)$ : Probability that an VD has the value $a_{i} . P\left(t_{j} \mid a_{i}\right)$ : The conditional probability that target has the value $t_{j}$ when a VD has a value $a_{i}$. Although this function depends on the correlation among the $\mathrm{VD}$ set $\left(\mathrm{C}_{\mathrm{AA}}\right)$ and the $\mathrm{VD}$ to the corresponding target $\left(\mathrm{C}_{\mathrm{AT}}\right)$, it succeeds in very special cases. In real database the evaluation function fails if any VD has only one value $\left(\mathrm{C}_{\mathrm{AT}}=0.0 \& \mathrm{C}_{\mathrm{AA}}=\right.$ undefined value $)$. So this paper introduces a unit step function, $\mathrm{U}$, to modify the evaluation functions as follows:

$E_{s_{-} \bmod }=U \cdot E_{s}$

where;

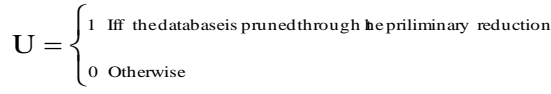

\subsubsection{Search strategy}

The number of $E_{\mathrm{s}}$ calculations to extract $\mathrm{U}_{\text {att_most }}$ from $\mathrm{U}_{\text {att_new }}$ which has $\mathrm{N}$ VDs is $\left(2^{N}-1\right)$. The search space increases exponentially with $\mathrm{N}$. This is computationally burdensome. A proposed methodology to extract $U_{\text {att_most }}$ and reduce the search space is presented in figure 4. The methodology is based on constructing $\mathrm{N}$ groups. The $\mathrm{i}^{\text {th }}$ group $\left(\mathrm{G}_{\mathrm{i}}\right)$ includes number of sets equal to $(N-i+1)$. The total number of sets required for the $\mathrm{E}_{\mathrm{s}}$ calculations will be $\sum_{i=1}^{i=N} N-i+1$. This

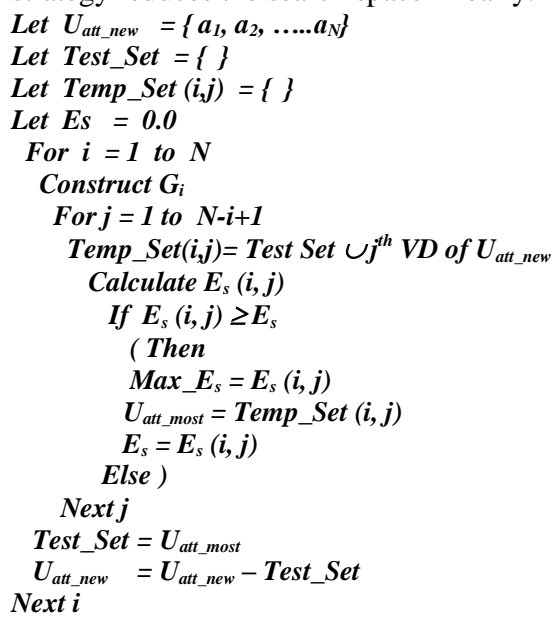

Fig 4: Search strategy for the selection of most relevant VDs.

\section{SIMILARITY MATCHING}

To compare the shape VDs, similarity distance metric is used as shown below:

$$
D(Q, T)=\sum_{i=1}^{l}\left|\frac{f_{q, i}-f_{t, i}}{1+f_{q, i}+f_{t, i}}\right|
$$

Where $\mathrm{Q}$ is query image, 1 is feature vector length, $\mathrm{T}$ is image in database; $f_{q, i}$ is $i^{t h}$ VD of query image Q, $f_{t, i}$ is $i^{t h}$ VD of image $\mathrm{T}$ in the database.

For the similarity calculation, to compare the color VD vectors of the query image $\mathrm{Q}$ and a target image $\mathrm{T}$ in the database, we used

$$
D(Q, T)=\sum_{k=1}^{l}\left|F_{q, k}-F_{t, k}\right|
$$

Where $F_{q, k}$ is the color VD vector of the k-th sub-block in image $\mathrm{Q}$ and $F_{t, k}$ is that of image T, 1 is the number of subblocks [24].

Comparing the edge feature histograms requires the distance $D(Q, T)$ of two image histograms $Q$ and $T$ using the following measure:

$$
\begin{aligned}
& \left.D(Q, T)=\sum_{i=0}^{4} \mid \operatorname{Avg}\left(\text { Local }_{-} Q[i]\right)-\operatorname{Avg}_{(\text {Local_}} T[i]\right) \mid \\
& \left.+5 \times \sum_{i=0}^{4} \mid \text { Global_Q} Q i\right]- \text { Global_}_{-}[i] \mid
\end{aligned}
$$

Where Local_Q[i] represents the reconstructed value of Bin_Count[i] of image $Q$ and Local_T[i] is that of image T [25]. Global_Q[] and Global_T[] represent the normalized bin values for the global edge histograms of image $Q$ and $T$, respectively. Since the number of bins of the global histogram is relatively smaller than that of local histogram, a weighting factor of 5 is applied in (27).

Let $D_{s}$ be the result of the difference between the query image and a database based on shape VDs, $D_{C}$ be the result of the difference between the query image and a database based on color VDs vectors and $D_{e}$ is the result of the difference between the query image and a database based on edge histogram, then the total difference take the form [26, 27]:

$$
D_{s c e}=\alpha \cdot D_{s}+\beta \cdot D_{c}+\gamma \cdot D_{e} \text {, where } \alpha+\beta+\gamma=1
$$

\section{EXPERIMENTAL RESULTS AND DISCUSSION}

A technique based on NS clustering algorithm to image segmentation is proposed. First, the image is transformed into NS based on T, I, F and a new adaptive threshold is employed to reduce the indeterminacy degree of the image, which is evaluated by the entropy of the indeterminate subset. NS image is enhanced using intensification transformation to improve the quality and emphasizes certain VDs of image to makes segmentation easier and more effective. The image becomes more uniform and homogenous, and more suitable for segmentation. Then, the image in NS domain passed to adaptive threshold for $\mathrm{T}$, I and $\mathrm{F}$.

The proposed approach was tested on 30 images and compared the performance with that of some existing methods such as classical fuzzy c-mean, local threshold and global threshold.

Figure 5 shows results of the proposed approach on different slice. The image is transformed from image domain to True 
domain objects as seen in Figure 5(b), Figure 5(c) shows the image transformed into False domain objects, Figure 5(d) use intensification transformation to enhance the image in the new domain, Figure 5(e, f) show the adaptive threshold for $\mathrm{T}$ image and F-image using FCM. In Figure $5(\mathrm{~g}, \mathrm{~h})$ define Indeterminate image from the homogeneity value of $\mathrm{T}$ using the absolute value of difference between intensity $g(i, j)$ and its local mean value, Figure 5(i) shows adaptive threshold for I-image using FCM. Figure 5(j) binary NS image based on T, I, F. Finally, the results of the proposed segmentation approach are shown in Figure 5(k, l).

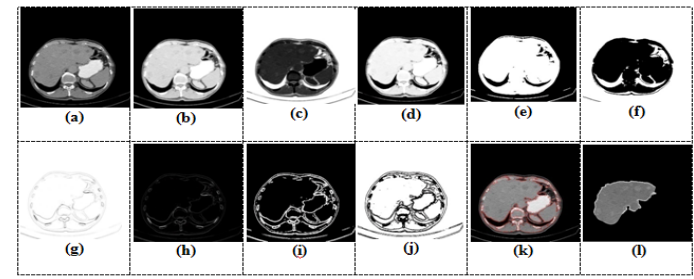

Fig 5: NS algorithm for Liver segmentation

Experiments were conducted using an image database consisting of Commercial Corel Image Gallery images [28]. Six images selected in the database were used to make query images and ground-truth images (the ground-truth data is a set of visually similar images for a given query image) are categorized by image classes based on the query images as shows in Fig. 6.

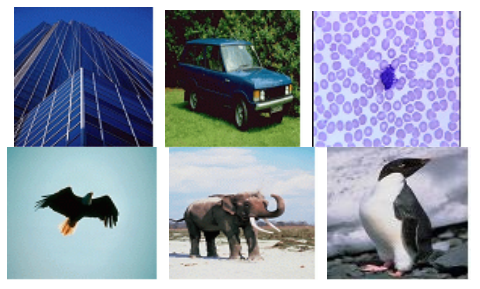

Fig 6: Query images.

The ground-truths that used in experiments were determined by three participants of the MPHG-7 CE. As a measure of retrieval accuracy, the Average Normalized Modified Retrieval Rank (ANMRR) is used [29]. Precision and Recall are well known measures for the retrieval performance. They are basically a "hit and miss" counter. That is, the performance is based on the number of retrieved images, which have similarity measures that are greater than a given threshold. For more specific comparisons, the rank information among the retrieved images in needed. ANMRR is the measure that exploits the rank of the retrieved images as well. Note that lower ANMRR values indicate more accurate retrieval performance. Table 2 shows the performance comparisons of NMRR and ANMRR.

Table 2. Retrieval performance

\begin{tabular}{llllll} 
& Shape & Color & Edges & \multicolumn{2}{c}{ All VDs M.R. VDs } \\
\cline { 2 - 5 } query1 & 0.8732 & 0.3502 & 0.9303 & 0.9032 & 0.4038 \\
query2 & 0.8156 & 0.8497 & 0.7602 & 0.6320 & 0.6403 \\
query3 & 0.9201 & 0.7498 & 0.4834 & 0.5302 & 0.6402 \\
query4 & 0.5289 & 0.6907 & 0.6424 & 0.7021 & 0.5498 \\
query5 & 0.4903 & 0.8503 & 0.7357 & 0.3203 & 0.7340 \\
query6 & 0.8335 & 0.8545 & 0.5632 & 0.4392 & 0.4530 \\
\hline ANMRR & 0.7436 & 0.7242 & 0.6858 & 0.5878 & 0.5701 \\
\hline
\end{tabular}

The first stage of experiment is concerned with all the VDs that can be extracted from the image as follows;
The GMs is used to extract the shape VDs that will be named in table 3 as $\left(V D_{1}, V D_{2}, \ldots, V D_{7}\right)$. In addition, the modified Stricker method on the HSI color space is applied to get the color VDs namely $\left(V D_{8}, V D_{9}, \ldots, V D_{12}\right)$. The MPEG-7 edge histogram descriptor is used to extract the edges VDs namely $\left(V D_{13}, V D_{14}, \ldots, V D_{17}\right)$. Finally, each image in the database has a VD vector that consists of 18 components; the last one is the class feature as shown in table 4 . Now, the second stage of experiment is performed to reduce the extracted VDs. This stage is represented by the CLIPS language which has strong search ability. It is beginning with fuzzification process to convert the continuous VDs to linguistic terms as shown in table 5. Table 6 shows the calculations of the correlation between VD-to-VD and VD-to-class and the corresponding evaluation functions are shown in table 7 . The most relevant VDs set includes $\left\{\mathbf{V D}_{\mathbf{9}}, \mathbf{V D}_{\mathbf{6}}, \mathbf{V D}_{\mathbf{1 3}}, \mathbf{V D}_{\mathbf{1 7}}\right\}$, which has the largest evaluation function value, $\mathrm{E}_{\mathrm{s}}=\mathbf{0 . 4 9 3}$.

The retrieval images in case of using all VDs extracting and the case of using the most relevant VDs for the same color query image are shown in figures 7 and 8 respectively. In case of presenting the query image to the system, the retrieval images quality is very good but the time consumed is much more.

The comparison between using all VDs and the most relevant VDs in the retrieved process is shown in Fig. 9. The results show that the average query time for returning 30 images is about $0.7 \mathrm{~s}$ for most relevant VDs and about $1.9 \mathrm{~s}$ for all VDs. This means that using the most relevant VDs reduce the calculation work and the computation time in the retrieval process. Then, it becomes clear that the retrieval time of the proposed model using the most relevant VDs is better than the using all VDs. The main advantage of these proposed algorithms is the balance between preservation of retrieval quality and consummation of retrieval time.

\section{CONCLUSION}

In this paper, a new images segmentation technique based on NS approach and an adaptive threshold has been proposed. The image is described as a NS using three membership sets $\mathrm{T}$, I and F. The entropy in NI domain is defined and employed to measure the indetermination. Adaptive threshold and NL are employed to obtain segmented image.

VDs for increasing the matching performance were presented. Three feature extraction methods based on shape, color and edges were proposed.

It must be emphasized that the main objective of this paper is increasing the retrieval performance, not only by using the VDs separately but also by using the all mentioned VDs to extract the all possible features from the same image. Consequently, we can deal with different kind of images that have shape, color and edges in the same time.

In addition, this paper introduces a novel technique for reducing a search space of evaluation function from exponential to linear function. Therefore, the time consumed for calculation of evaluation function is reduced. The overall results of using most relevant VDs in image retrieval satisfied the balance between preservation of retrieval quality and consummation of retrieval time.

\section{REFERENCES}

[1] Guoping Qiu et al., Visual guided navigation for image retrieval, Pattern Recognition 40(6), 2007, 1711-1721.

[2] V.P. Subramanyam, S.K. Sett, Knowledge-based image retrieval system, Knowledge-Based Systems 21(2), 2008, 89-100. 
[3] Veltkamp R. C., Tanase M.: Content-Based Image Retrieval Systems: A Survey. Revised and extended version of Technical Report UU-CS-2000-34, Department of Computing Science, Utrecht University, 2002.

[4] F. Smarandache,"A Unifying Field in Logics Neutrosophic Logic". Neutrosophy, Neutrosophic Set, Neutrosophic Probability, third ed: American Research Press, 2003.

[5] H. D. Cheng, Y. guot, and y. zhang, A novel image segmentation approach based on neutrosophic set and improved fuzzy c-means algorithm, world scientific publishing company, new math. and natural computation, vol. 7, no.1, 155-171, 2011.

[6] J. Mohan, V. Krishnaveni, Y. Guo, A new neutrosophic approach of wiener Filtering for MRI denoising, measurement science review, Vol. 13, No. 4, 177-186, 2013

[7] S.L. Phung, Abdesselam Bouzerdoum, 2007: Detecting people in images: An Edge Density Approach, IEEE, ICASSP, 1229-1232.

[8] S. Min, S. Park, and C. Won, "Image Retrieval via Query-by- Layout using MPEG-7 Visual Descriptors" ETRI Journal, Vol. 29, No. 2, 2007.

[9] M. Eom, Y. Choe, "Fast Extraction of Edge Histogram in DCT Domain based on MPEG-7" PWASET Vol. 9, 2005.

[10] J. Wei, E. Guihua, D. Qionghai, G. Jinwei, Similarity online feature selection in content-based image retrieval, IEEE Transactions on Image Processing 15(3), 2006, $702-712$.

[11] F. Smarandache, "A Unifying Field in Logics Neutrosophic Logic". Neutrosophy, Neutrosophic Set, Neutrosophic Probability, third ed: American Research Press, 2003.

[12] Ming Zhang, Ling Zhang, H.D. Cheng, "A neutrosophic approach to image segmentation based on watershed method", Elsevier Signal Processing 90, 1510-1517, 2010.

[13] H. D. Cheng, Y. Guot, Y. Zhang, "A Novel Image Segmentation Approach Based on Neutrosophic Set And Improved Fuzzy C-Means Algorithm", World Scientific Publishing Company, New Math. and Natural Computation, Vol. 7, No.1, 155-171, 2011.

[14] Y. Guo, H.D. Cheng, Y. Zhang, W. Zhao, "A new neutrosophic appraoch to image thresholding", Atlantis Press, Proceedings of the 11 th Joint Conference on Information Sciences, 1-6, 2008.

[15] A. Ahirwar, "Study of Techniques used for Medical Image Segmentation and Computation of Statistical Test for Region Classification of Brain MRI", I. J. Information Technology and Computer Science, 05, 4453,2013

[16] Preeti Aggarwal, H.K. Sardana, Gagandeep Jindal, Content-Based Medical Image Retrieval: Theory, Gaps and Future Directions, ICGST-GVIP Journal, Vol. 9, Issue (II), April 2009.
[17] M. J. Swain and D. H. Ballard, "Color Indexing", International Journal of Computer Vision, Vol. 7, No. 1, 11-32, 1991.

[18] B. Funt and G. Finlayson, "Color Constant Color Indexing" IEEE Trans. Pattern Analysis and Machine Intelligence, Vol. 17, No. 5, 522-529, 1995.

[19] M. Stricker and M. Orengo, "Similarity of color Images" SPIE, Vol. 220, No. 1, 381-392, 1995.

[20] B. S. Manjunath, P. Salembier, T. Sikora, "Introduction to MPEG-7", JOHN WILLEY\&SONS, LTD, 183-184, 2002.

[21] W. Pedrycz and George Vukovich "Feature analysis through information granulation and fuzzy sets" ARTICLE Pattern Recognition, Volume 35, Issue 4, April 2002, 825-834.

[22] M. A. Berry and Gordon Linoff, "Mastering Data Mining the Art and Science of Customer Relationship Management" Wiley computer publishing, John Wiley \& Sons, Inc. 2000.

[23] R. M. Hogarth. "Methods for Aggregating Opinions", In H. Jungermann and G. De Zeeuw, Editors, Decision Making and Change in Human Affairs. D. Reidel Publishing, Dordrecht-Holland, 1977.

[24] C. Salton and C. Buckley "Improving Retrieval Performance by Relevance Feedback" the research report of Cornell University, 1-24, 1988.

[25] C. Won, D. Park, and S. Jun, "Efficient Use of MPEG-7 Edge Histogram Descriptor" ETRI Journal, Vol. 24, No. $1,2002$.

[26] Mohamed Eisa, Ibrahim El-Henawy, A. E. Elalfi and Hans Burkhardt "Image Retrieval based on Invariant Features and Histogram Refinement" ICGST International Conference on Graphics, Vision and Image Processing, GVIP 05 Conference, 19-21 Dec. 2005, CICC, Cairo, Egypt.

[27] Ibrahim El-Henawy, Mohamed Eisa, A. E. Elalfi and Hans Burkhardt "Image Retrieval using Local Color and Texture Features" ICGST International Journal on Graphics, Vision and Image Processing (GVIP), May. 2005, Vol. SI1, Pages 47-52.

[28] Corel Corporation. URL: http://www.corel.com/ (2005)

[29] ISO/IEC/JTC1/SC29/WG11: "Description of Core Experiments for MPEG-7 Color/Texture Descriptors," MPEG document N2929, Melbourne, 1999.
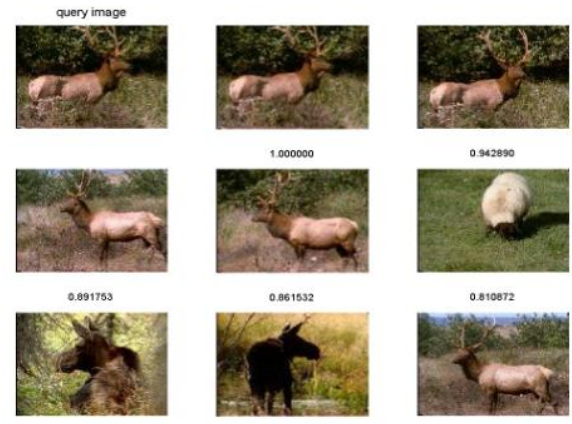

0.909327

0750012

0710263

Fig 7: The retrieved images using all VDs. 


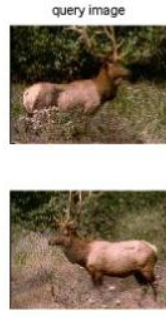

0.91753

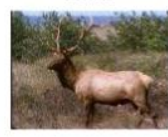

0.800327

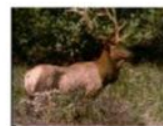

1.000000

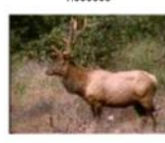

0.941532

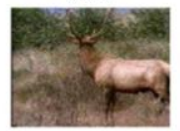

0.873212

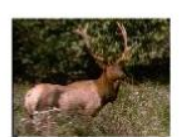

0.982990

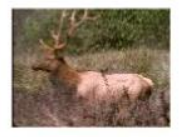

0.910932

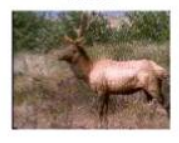

0.853760
Fig 8: The retrieved images using most relevant VDs.

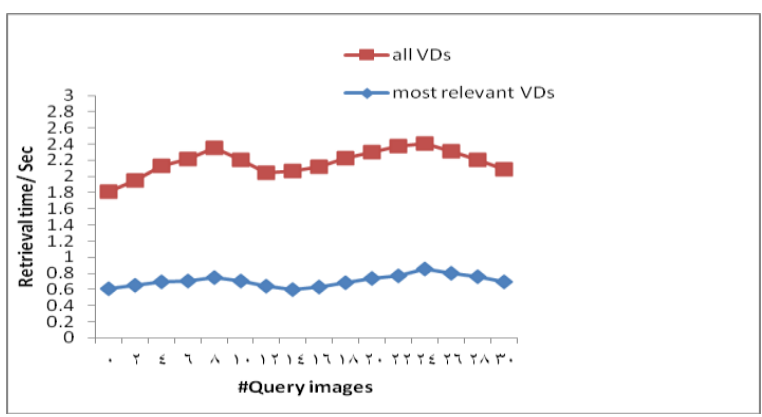

Fig 9: Comparison between the times consumed in two cases.

Table 7. Evaluation Function for all Possible Sets

\begin{tabular}{|c|c|c|c|}
\hline Groups & Possible Sets & $\mathbf{E}_{\mathbf{s}_{-} \text {mod }}$ & $\begin{array}{c}\text { Max. } E_{\text {s-mod }} \\
\text { in each group }\end{array}$ \\
\hline \multirow{13}{*}{$\mathrm{G}_{1}$} & $\left\{\mathrm{VD}_{1}\right\}$ & 0.116 & \multirow{13}{*}{0.273} \\
\hline & $\left\{\mathrm{VD}_{3}\right\}$ & 0.107 & \\
\hline & $\left\{\mathrm{VD}_{4}\right\}$ & 0.195 & \\
\hline & $\left\{\mathrm{VD}_{6}\right\}$ & 0.245 & \\
\hline & $\left\{\mathrm{VD}_{8}\right\}$ & 0.109 & \\
\hline & $\left\{\mathbf{V D}_{\mathbf{9}}\right\}$ & 0.273 & \\
\hline & $\left\{\mathrm{VD}_{10}\right\}$ & 0.218 & \\
\hline & $\left\{\mathrm{VD}_{11}\right\}$ & 0.206 & \\
\hline & $\left\{\mathrm{VD}_{12}\right\}$ & 0.174 & \\
\hline & $\left\{\mathrm{VD}_{13}\right\}$ & 0.129 & \\
\hline & $\left\{\mathrm{VD}_{14}\right\}$ & 0.204 & \\
\hline & $\left\{\mathrm{VD}_{16}\right\}$ & 0.095 & \\
\hline & $\left\{\mathrm{VD}_{17}\right\}$ & 0.211 & \\
\hline
\end{tabular}

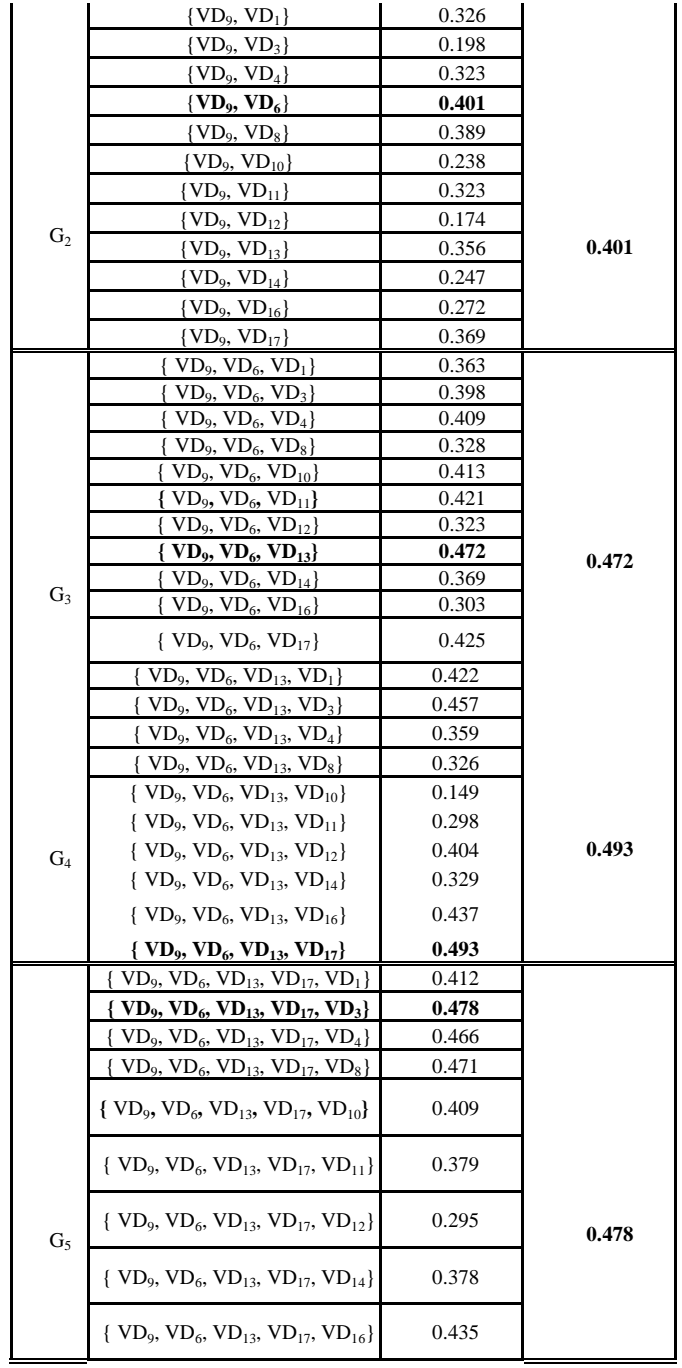

Table 3. The VDs from image database

\begin{tabular}{ccc}
\hline & Visual descriptors & \\
\hline Shape descriptor & Color descriptor & Edge descriptor \\
\hline Geometrical moments & Stricker method & MPEG-7 \\
\hline RGB channels & HSI channels & RGB channels \\
\hline
\end{tabular}

$\begin{array}{llllllllllllllllllll}M_{1} & M_{2} & M_{3} & M_{4} & M_{5} & M_{6} & M_{7} & E_{H} & \delta_{H} & \alpha_{H} & E_{S} & E_{I} & \text { Local ( average of } 16 \text { sub-images ) }\end{array}$

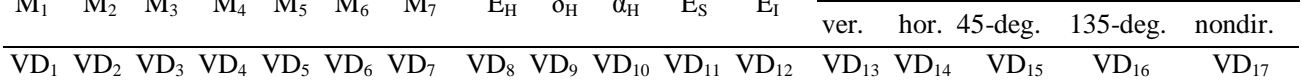

Table 4. The Sample of The original VDs Extracted from Image Database.

\begin{tabular}{|c|c|c|c|c|c|c|c|c|c|c|c|c|c|c|c|c|c|c|}
\hline Images & $\mathrm{VD}_{1}$ & $\mathrm{VD}_{2}$ & $\mathrm{VD}_{3}$ & $\mathrm{VD}_{4}$ & $\mathrm{VD}_{5}$ & $\mathrm{VD}_{6}$ & $\mathrm{VD}_{7}$ & $\mathrm{VD}_{8}$ & $\mathrm{VD}_{9}$ & $\mathrm{VD}_{10}$ & $\mathrm{VD}_{11}$ & $\mathrm{VD}_{12}$ & \begin{tabular}{|l|}
$\mathrm{VD}_{13}$ \\
\end{tabular} & $\mathrm{VD}_{14}$ & $\mathrm{VD}_{15}$ & $\mathrm{VD}_{16}$ & $\mathrm{VD}_{17}$ & CLASS \\
\hline img 1 & 310 & 030 & 590 & 360 & 5.457 & 006 & 6915.7 & 4.8 & 2.40 & 264 & & 67.1 & 16.49 & 4.56 & 5.49 & 14.23 & 3.24 & $\begin{array}{ll}\mathrm{C} 1 \\
\end{array}$ \\
\hline \begin{tabular}{|l|}
$\operatorname{img} 2$ \\
\end{tabular} & 64.210 & 5.480 & 173.900 & 5.270 & 164.852 & 264.846 & \begin{tabular}{|l|}
6777.8 \\
\end{tabular} & 6940.3 & 6792.4 & 5167.13 & 6704.35 & 6197.4 & 6.59 & 9.458 & 13.4 & 6.48 & 15.49 & $\mathrm{C} 3$ \\
\hline \begin{tabular}{|l|} 
img 3 \\
\end{tabular} & 48.180 & 112.500 & 4.560 & 6.068 & \begin{tabular}{|l|}
49.512 \\
\end{tabular} & 194.843 & 7089.22 & 6597.86 & 6711.5 & 9324.61 & 9230.45 & 10947.6 & 20.49 & \begin{tabular}{|l|}
11.45 \\
\end{tabular} & 9.05 & 4.49 & 7.78 & C4 \\
\hline \begin{tabular}{|l|} 
img 4 \\
\end{tabular} & 21.810 & 297.000 & 169.900 & 2.355 & \begin{tabular}{|l|}
59.486 \\
\end{tabular} & 186.772 & 7109.8 & 6694.5 & 6943.57 & \begin{tabular}{|l|}
4557.6 \\
\end{tabular} & 5006.94 & 4879.3 & \begin{tabular}{|l|l}
7.89 \\
\end{tabular} & 4.885 & 17.6 & 10.06 & 6.7 & C5 \\
\hline img 5 & 63.900 & 1.500 & 67.680 & 106.555 & 88.492 & 115.975 & 6798.08 & 7110.54 & 7952.4 & 5519.04 & 6790.05 & 1648.7 & \begin{tabular}{|l|}
19.4 \\
\end{tabular} & 12.95 & 8.173 & 10.15 & 9 & $\mathrm{C} 2$ \\
\hline \begin{tabular}{|l|} 
img 6 \\
\end{tabular} & 68.780 & 190.590 & 227.590 & 246.794 & 166.482 & 261.005 & \begin{tabular}{|l|}
6883.12 \\
\end{tabular} & 7264.9 & \begin{tabular}{|l|}
7782.4 \\
\end{tabular} & 6899.41 & 7910.50 & 5648.9 & 8.05 & 5.789 & 12.645 & 9.08 & 6 & C5 \\
\hline img 7 & 321.400 & 310.840 & 124.120 & 482.474 & 148.799 & 483.795 & 7245.8 & 6994.58 & 6882.04 & \begin{tabular}{|l|}
5778.2 \\
\end{tabular} & 10648.51 & \begin{tabular}{|l|}
10549.2 \\
\end{tabular} & \begin{tabular}{|l|l}
17.8 \\
\end{tabular} & 10.346 & 10.04 & 16.49 & 7.44 & C1 \\
\hline img 8 & 465.120 & 387.870 & 146.870 & 444.197 & 283.310 & 433.277 & 6895 & 6999 & 6997.85 & 5123.8 & 5648.81 & 6328.2 & 6.6 & 7.99 & 4.01 & 15.4 & 7.5 & C2 \\
\hline \begin{tabular}{|l|}
$\operatorname{img} 9$ \\
\end{tabular} & 64.780 & 215.000 & \begin{tabular}{|l|l|}
69.590 \\
\end{tabular} & 201.495 & 164.855 & 264.849 & 6773.2 & 6789.11 & 6799.8 & 5997.5 & 5521.89 & 4618 & 7.12 & 4.895 & 13.84 & 6.1 & 6.4 & C5 \\
\hline Img10 & 117.430 & 141.590 & \begin{tabular}{|l|}
65.120 \\
\end{tabular} & 315.264 & \begin{tabular}{|l|}
46.057 \\
\end{tabular} & 164.997 & \begin{tabular}{|l|}
7169.47 \\
\end{tabular} & 6498.7 & 6700.4 & 5468.7 & 7168.49 & 8947.1 & 6.03 & 7.68 & 13.4 & 4.8 & 5.1 & C4 \\
\hline g11 & 46.870 & 2.090 & 4.960 & 264.710 & 158.645 & 264.879 & 7200.6 & 7340.45 & 6804.5 & 7259.8 & 7923.46 & 391.4 & 15.3 & 13.49 & 2.604 & 8.06 & 12.4 & C3 \\
\hline
\end{tabular}




\begin{tabular}{|c|c|c|c|c|c|c|c|c|c|c|c|c|c|c|c|c|c|c|}
\hline ng12 & 1.460 & 1.680 & \begin{tabular}{|l|}
169.090 \\
\end{tabular} & 1.005 & 264.879 & 264.851 & 6800.7 & \begin{tabular}{|l|}
7110.56 \\
\end{tabular} & 7699.58 & \begin{tabular}{|l|}
3987.5 \\
\end{tabular} & 4978.56 & 4687.9 & 4.9 & 6.48 & 16.49 & 7.07 & 1.7 & $\mathrm{C5}$ \\
\hline Img13 & .750 & 5.750 & 146.500 & 3.594 & 164.003 & 260.495 & 6977.24 & 7214.801 & 7881.11 & 7648.23 & 7982.45 & 7892.4 & 16.2 & 13.49 & 8.06 & 11.64 & 9.82 & C2 \\
\hline ng14 & 6.250 & 2.060 & 3.500 & 4.266 & 94.672 & 6.489 & 01.54 & 6889.7 & 7752.95 & 7536.48 & 10648.7 & \begin{tabular}{l|l|}
776.4 \\
\end{tabular} & 6.19 & 3.55 & 7.45 & 8.57 & 5.1 & C1 \\
\hline ng15 & 5.870 & 4.430 & 7.930 & 2.108 & 41.640 & 264.847 & 7025.4 & 6924.1 & \begin{tabular}{|l|l|}
7951.8 \\
\end{tabular} & 6387.9 & 891.5 & 984.1 & \begin{tabular}{|l|}
7.94 \\
\end{tabular} & .66 & 4.106 & 6.15 & 6.19 & C3 \\
\hline 16 & 80.590 & D. & $1-2$ & 70 & 8 & 9 & 7164.89 & 6832.5 & 7996.3 & 5978.4 & 894.6 & 9.5 & 8.4 & .84 & 5.49 & $12.4 \mathrm{~S}$ & .7 & C4 \\
\hline g17 & .150 & 10 & 20 & 700 & 812 & 35 & 4.6 & 23.7 & 16 & 5798.4 & & 3 & 16.40 & 46 & 101 & 6.08 & 4 & C3 \\
\hline g18 & 120 & 308.960 & 102.780 & 5.550 & 880 & .646 & 0.6 & .3 & 82.45 & 5134.7 & 5814.6 & 9.4 & 7.4 & 0.64 & 3.85 & 9.66 & .7 & $\overline{C 2}$ \\
\hline $\mathrm{ng} 19$ & 3.210 & 46.870 & 287.930 & 3.202 & 248.997 & 364.300 & 7160.5 & 6710.5 & 6945.8 & 5463.79 & 10648.3 & 10459 & 14 & 3.55 & 9.84 & 11.86 & 14.2 & C1 \\
\hline ng20 & 57.560 & \begin{tabular}{|l|l|}
404.120 \\
\end{tabular} & 47.340 & 54.870 & 78.940 & 211.600 & 6934.4 & 7135 & \begin{tabular}{|l|}
7943.5 \\
\end{tabular} & 5312.8 & 5782.4 & 5178.3 & \begin{tabular}{|l|}
8.43 \\
\end{tabular} & 6.43 & 10.06 & 10.1 & 11.29 & C5 \\
\hline Img21 & 391.930 & 178.650 & 99.590 & 199.164 & 84.156 & 180.946 & 6923.4 & 7199.5 & 7994.02 & 7258.9 & 9849.9 & 10336 & \begin{tabular}{|l|}
14.22 \\
\end{tabular} & 7.53 & 6.012 & 9.5 & 8.99 & C1 \\
\hline $\operatorname{Img} 22$ & 190.960 & 409.210 & 186.150 & 1.111 & 493.682 & 487.905 & 6798.33 & \begin{tabular}{|l|l|}
7345.87 \\
\end{tabular} & 7855.3 & 11264.8 & 9315.7 & 10648 & 8.31 & 9.41 & 13.8 & 4.6 & 6.8 & C4 \\
\hline $\operatorname{Img} 23$ & 89.960 & 370.400 & 217.530 & 3.645 & 184.205 & 266.844 & 6778.10 & 6890.5 & 6798.03 & 6197.8 & 5978.4 & 5197.5 & 5.99 & 8.06 & 18.9 & 10.06 & 9.02 & C5 \\
\hline Img24 & 82.210 & 442.400 & 78.500 & 208.741 & 264.815 & 297.643 & 6990.57 & 6952.01 & 6995.78 & 5138.79 & 5187.3 & 5003.4 & 8.37 & 9.7 & 17.56 & 13.54 & 14.8 & C4 \\
\hline Img25 & 118.680 & \begin{tabular}{|l|l|}
407.030 \\
\end{tabular} & 112.500 & 1.488 & 179.852 & 225.788 & 6889.4 & 6792.3 & 7780.45 & 5679.22 & 6498.99 & 6158.9 & \begin{tabular}{|l|}
14.6 \\
\end{tabular} & 10.05 & 12.06 & 9.54 & 12.6 & $\mathrm{C} 2$ \\
\hline $\lg 26$ & 870 & 5.120 & 870 & 4.211 & 56.481 & 140.945 & 6943.8 & 0.6 & \begin{tabular}{|l|l|}
7958.4 \\
\end{tabular} & 5137.94 & 6197.8 & 6489.7 & \begin{tabular}{|l|}
16.4 \\
\end{tabular} & 7.44 & 10.24 & 6.83 & 16.05 & C4 \\
\hline $\mathrm{g} 27$ & 3.650 & 2.400 & 83.620 & 9.992 & 201.584 & 287.664 & 6794.01 & 6993.5 & 6789.13 & 5341.89 & 5123.7 & 6258.1 & 18.45 & 13.06 & 10.64 & 4.4 & 15.8 & $\mathrm{C3}$ \\
\hline g28 & 2.810 & 3.340 & 399.870 & 9.180 & 64.852 & 189.004 & 6694.15 & 7245.8 & 6891.06 & 7564.8 & 431.5 & 9548.3 & 10.94 & 4.85 & 6.48 & 12.8 & 16.4 & C1 \\
\hline ig29 & .060 & 0.500 & 46.870 & 0.461 & 79.209 & 55.443 & 6794.5 & 7154.8 & 6990.5 & 7928.3 & 5132.4 & 7497.5 & \begin{tabular}{|l|}
9.84 \\
\end{tabular} & 6.11 & 7.92 & 14.09 & 11 & $\mathrm{C} 2$ \\
\hline $\mathrm{ng} 30$ & 34.400 & 65.120 & 206.250 & 57.159 & 176.843 & 267.999 & \begin{tabular}{|l|}
6832.4 \\
\end{tabular} & 264 & 883.09 & 584 & 5978.12 & 7234.6 & 7.8 & 3.01 & .84 & 3.49 & 7.4 & $\mathrm{C3}$ \\
\hline
\end{tabular}

\section{Table 5. The fuzzy values of VDs extracted from Images Database}

\begin{tabular}{|c|c|c|c|c|c|c|c|c|c|c|c|c|c|c|c|c|c|c|}
\hline ages & $D_{1}$ & $D_{2}$ & $\mathbf{D}_{3}$ & $D_{4}$ & $D_{5}$ & $D_{6}$ & $\mathbf{D}_{7}$ & $\mathbf{D}_{8}$ & 9 & 10 & 11 & $V D_{12}$ & $V D_{13}$ & 14 & $V_{15}$ & $D_{16}$ & $D_{17}$ & \\
\hline & High & jigh & High & & Low & dium & High & Medium & Medium & & & & & & & & & C1 \\
\hline & & & & & w & & & & & & gh & & & & $\mathrm{w}$ & & & C3 \\
\hline g 3 & ledium & High & Mlu & igh & Low & igh & gh & Medium & & igh & Low & High & Iedium & edium & Low & Medium & Edum & Y \\
\hline $\lg 4$ & edium & High & Medium & High & Low & High & igh & High & High & Medium & Medium & Low & High & lgn & Low & $11 \mathrm{gh}$ & ledium & $C_{5}$ \\
\hline ng 5 & Low & High & Low & High & Low & High & High & Medium & Low & edium & High & Low & Medium & Medium & Low & Medium & High & C2 \\
\hline ng 6 & Low & High & & High & Low & High & igh & & $\mathrm{Hig}$ & $\mathrm{m}$ & Low & Medium & High & High & Low & um & $\mathrm{H}$ & 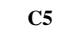 \\
\hline $\lg 7$ & $\mathrm{H}$ & igh & High & & Low & & $\mathrm{H}$ & & H & & $\mathrm{H}$ & & & & w & & & C1 \\
\hline $\lg 8$ & High & igh & High & & Low & $\mathrm{M}$ & High & $\mathrm{Mec}$ & Medium & Medium & $\mathrm{Med}$ & $\mathrm{Mec}$ & $\mathrm{Me}$ & igh & Low & & & 2 \\
\hline g 9 & L & & & $\mathrm{H}$ & Low & High & igh & & & & $\mathrm{Me}$ & L & & & & & & 5 \\
\hline g 10 & L & & & & Low & & & & & & & $\mathrm{H}$ & & & WW & & & 8 \\
\hline g 11 & Lo & & $\mathrm{L}$ & & Low & & & & & & $\mathrm{H}$ & 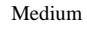 & $\mathrm{n}$ & & Low & & & 3 \\
\hline g 12 & Low & 1. & $\mathrm{~L}$ & & Low & igh & 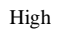 & & & & $\mathrm{Hi}$ & Loy & & & Low & & & 5 \\
\hline g 13 & Low & High & $\mathrm{L}$ & & Low & High & High & $\mathrm{m}$ & III & $\mathrm{m}$ & Low & Med & $\mathrm{m}$ & $\mathrm{M}$ & Low & $\mathrm{N}$ & & 2 \\
\hline g 14 & Medium & High & Med & hh & Low & High & High & & $\mathrm{Hi}$ & High & $\mathrm{m}$ & High & & & Low & & & $\mathrm{C} 1$ \\
\hline g 15 & Jow & High & Low & Hiop & Low & lish & Hi & & & $\mathrm{m}$ & Low & $\mathrm{Mec}$ & High & & Low & & & $\mathrm{c3}$ \\
\hline g 16 & Low & High & & & Low & igh & High & & & $\mathrm{m}$ & Medium & $\mathrm{Med}$ & & & Low & & & 4 \\
\hline g 17 & Mediu & High & $\mathrm{Me}$ & High & Low & $H$ & $\mathrm{Hi}$ & & & edium & $\mathrm{L}$ & Med & 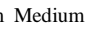 & n & Low & 1 & & 3 \\
\hline $\mathrm{g} 18$ & & High & & Jjoh & Low & $H$ & High & & $\mathrm{m}$ & edium & Medium & $\mathrm{Im}$ & & & Low & & & 2 \\
\hline g 19 & High & $\mathrm{H}$ & & & Low & & $\mathrm{Hig}$ & & & & $\mathrm{Med}$ & & & $\mathrm{Im}$ & Low & & & 1 \\
\hline g 20 & Low & High & $\mathrm{L}$ & h & Low & $\mathrm{H}$ & $\mathrm{Hig}$ & 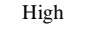 & & $\mathrm{M}$ & Med & $\mathrm{Me}$ & & 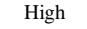 & on & & & 5 \\
\hline 21 & 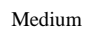 & $\mathrm{Hig}$ & Yedium & oh & Low & $\mathrm{Hi}$ & $\mathrm{High}$ & $\mathrm{Mec}$ & $\mathrm{Mec}$ & $\mathrm{M}$ & L & High & IIII & & $\mathrm{L}$ & & & \\
\hline & & High & Ied & dium & Low & Medi & High & & & 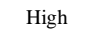 & $\mathrm{Mec}$ & & & $\mathrm{M}$ & Low & & & 4 \\
\hline g 23 & Low & $\mathrm{His}$ & & & Low & & sh & & & $\mathrm{m}$ & & & & & ow & & & 5 \\
\hline g 24 & Low & High & & & Low & igh & High & igh & Low & dium & High & M & High & igh & ow & igh & gh & 4 \\
\hline g 25 & Low & High & & & Low & ligh & High & Medium & Low & Medium & Medium & Medium & High & igh & Low & ligh & 511 & $\mathrm{C} 2$ \\
\hline g 26 & Low & High & ow & & Low & h & High & High & Medium & Medium & High & Medium & Medium & Medium & Low & Medium & $\mathrm{gh}$ & $\mathrm{C} 4$ \\
\hline & & & & & Low & & High & $\mathrm{Hi}$ & Medium & Medium & High & Medium & High & Medium & Low & Medium & SII & 3 \\
\hline & & & & & LO & & & & & ium & Medium & High & & igh & Low & & & 1 \\
\hline & & & & & Loy & & & & & lium & & dium & & & Low & & & 2 \\
\hline g 30 & diur & High & & igh & Low & High & High & High & ediun & edium & diun & ediu & Iediun & Medium & Low & Tedium & High & $\mathrm{C} 3$ \\
\hline
\end{tabular}

\section{Table 6. The Correlation Matrix for VDs extracted from images Database}

\begin{tabular}{l|c|c|c|c|c|c|c|c|c|c|c|c|c|c|} 
& $\mathrm{VD}_{1}$ & $\mathrm{VD}_{3}$ & $\mathrm{VD}_{4}$ & $\mathrm{VD}_{6}$ & $\mathrm{VD}_{8}$ & $\mathrm{VD}_{9}$ & $\mathrm{VD}_{10}$ & $\mathrm{VD}_{11}$ & $\mathrm{VD}_{12}$ & $\mathrm{VD}_{13}$ & $\mathrm{VD}_{14}$ & $\mathrm{VD}_{16}$ & $\mathrm{VD}_{17}$ & $\mathrm{CLASS}$ \\
\hline $\mathrm{VD}_{9}$ & 0.7510 & 0.6001 & 0.8900 & 1.0000 & 0.8940 & 0.6497 & 0.7012 & 0.4332 & 0.5862 & 0.5648 & 0.6240 & 0.5524 & 0.5116 & 0.8552 \\
\hline $\mathrm{VD}_{6}$ & 0.9962 & 0.8412 & 0.1487 & 0.0825 & 0.7459 & 0.0034 & 0.8463 & 1.0000 & 0.5147 & 0.5410 & 0.7847 & 0.5578 & 0.6954 & 0.7741 \\
\hline $\mathrm{VD}_{13}$ & 0.6483 & 0.5510 & 1.0000 & 0.7391 & 0.8546 & 0.7123 & 0.4216 & 0.7231 & 0.5369 & 0.5647 & 0.5857 & 0.5001 & 0.7104 & 0.5140 \\
\hline $\mathrm{VD}_{4}$ & 0.5140 & 0.6843 & 0.5379 & 0.5852 & 0.5548 & 1.0000 & 0.5564 & 0.5943 & 0.9137 & 0.0158 & 0.7948 & 0.1134 & 0.7510 & 0.7543 \\
\hline $\mathrm{VD}_{11}$ & 0.6783 & 0.7994 & 0.6248 & 0.7314 & 0.6702 & 0.7985 & 0.6402 & 0.5510 & 1.0000 & 0.7842 & 0.8699 & 0.8510 & 0.2445 & 0.5543 \\
\hline $\mathrm{VD}_{3}$ & 1.0000 & 0.5548 & 0.1597 & 0.5454 & 0.5648 & 0.5972 & 0.5064 & 0.5180 & 0.6482 & 0.8942 & 0.6215 & 0.0031 & 0.8010 & 0.5697 \\
\hline $\mathrm{VD}_{16}$ & 0.06482 & 0.0644 & 0.0258 & 0.9489 & 1.0000 & 0.4450 & 0.7951 & 0.6325 & 0.6284 & 0.5784 & 0.7514 & 0.5671 & 0.7014 & 0.5114 \\
\hline $\mathrm{VD}_{17}$ & 0.5997 & 1.0000 & 0.5317 & 0.5096 & 0.5538 & 0.5316 & 0.5564 & 0.5007 & 0.7619 & 0.1549 & 0.6110 & 0.3451 & 0.8992 & 0.5564 \\
\hline $\mathrm{VD}_{10}$ & 0.1648 & 0.0603 & 0.1679 & 0.0348 & 0.1034 & 0.0777 & 0.8811 & 0.8624 & 0.6391 & 0.5874 & 0.7749 & 0.5613 & 1.0000 & 0.6691 \\
\hline $\mathrm{VD}_{8}$ & 0.9871 & 0.0451 & 0.0369 & 0.4486 & 0.0357 & 0.6485 & 0.7059 & 0.6532 & 0.6471 & 1.0000 & 0.7841 & 0.5518 & 0.7712 & 0.5005 \\
\hline $\mathrm{VD}_{14}$ & 0.0064 & 0.0311 & 0.1473 & 0.9654 & 0.9872 & 0.9745 & 0.6631 & 0.7845 & 0.6825 & 0.5601 & 0.7521 & 1.0000 & 0.6502 & 0.6615 \\
\hline $\mathrm{VD}_{1}$ & 0.7748 & 0.7812 & 0.7619 & 0.7543 & 0.7631 & 0.7162 & 0.5610 & 0.6982 & 0.8246 & 0.7787 & 0.1664 & 0.6481 & 0.8221 & 0.6894 \\
\hline $\mathrm{VD}_{12}$ & 0.0648 & 0.1664 & 0.00649 & 0.0946 & 0.8139 & 0.0331 & 0.5009 & 0.6321 & 0.7936 & 0.6624 & 1.0000 & 0.5540 & 0.7541 & 0.6318 \\
\hline
\end{tabular}

